\title{
大环内酯类免疫抑制剂他克莫司的生物合成机制研究进展
}

\author{
陈单丹 $a, b$ 岑沛霖 ${ }^{a}$ 刘 文 ${ }^{b}$ 徐志南*,a \\ ( ${ }^{a}$ 浙江大学化工系生物工程研究所 杭州 310027) \\ $\left({ }^{b}\right.$ 中国科学院上海有机化学研究所 生命有机国家重点实验室 上海 200032)
}

\begin{abstract}
摘要 他克莫司(FK506)是一种来源于土壤链霓菌的大环内酯类免疫抑制剂, 由典型的聚酮合酶(PKS)-非核糖体肽合 成酶(NRPS)杂合系统负责催化其生物合成. 他克莫司的化学结构特殊, 包括骨架环的哌啶单元、4-羊基-3-甲氧基环己 基官能团, 以及甲氧基和烯丙基侧链. 近年来, 关于他克莫司的生物合成机制, 特别是其特殊前体的形成途径的研究 发展迅速. 对他克莫司生物合成的酶学基础进行了系统性地综述, 重点总结了其前体形成机制的研究新进展. 关键词＼cjkstart他克莫司; FK506; 生物合成; 聚酮合酶; 非核糖体肽合成酶; 前体; 途径特异性
\end{abstract}

\section{Progress in Studying the Biosynthetic Mechanism of the Macrolide Immunosuppressant Tacrolimus}

\author{
Chen, Dandan ${ }^{a, b} \quad$ Cen, Peilin $^{a} \quad$ Liu, Wen $^{b} \quad$ Xu, Zhinan ${ }^{*, a}$ \\ $\left({ }^{a}\right.$ Department of Chemical and Biological Engineering, Zhejiang University, Hangzhou 310027) \\ ( ${ }^{b}$ State Key Laboratory of Bioorganic and Natural Products Chemistry, Shanghai Institute of Organic Chemistry, \\ Chinese Academy of Science, Shanghai 200032)
}

\begin{abstract}
Tacrolimus (FK506), derived from various soil Streptomyces species, is a potent macrolide immunosuppressant that is assembled by a typical polyketide synthase (PKS)-nonribosomal peptide synthetase (NRPS) hybrid system. Its chemical structure is unique, with a piperidine unit consisting in the polyketide skeleton, a 4-methoxy, 5-hydroxy-cyclohexyl functional group, as well as methoxyl and allyl side chains. Recently, studies on the biosynthetic mechanism of tacrolimus, particularly for the pathways encoding the unusual precursors, achieved great successes. In this review, we summarize the enzymatic basis in correspondence to the biosynthesis of tacrolimus, and emphasize on the recent progress in understanding the biosynthetic pathways of the unusual precursors 4,5-dihydroxycyclohex-1-enecarboxylic acid (DHCHC), methoxymalonyl-acyl carrier protein (ACP), allylmalonyl-coenzyme A (CoA) and $L$-pipecolic acid.
\end{abstract}

Keywords tacrolimus; FK506; biosynthesis; polyketide synthase (PKS); nonribosomal peptide synthetase (NRPS); precursor; pathway-specific

他克莫司(Tacrolimus, FK506)是一种二十三元大环 内酯类化合物, 由日本藤泽制药公司于 1981 年首次从 筑波链霉菌 Streptomyces tsukubaensis No. 9993 的代谢 产物中提取获得 ${ }^{[1]}$. 他克莫司能与受体蛋白 FKBP12 形 成复合体, 通过特异地与钙调磷酸酶紧密结合, 有效遏 制其肽脯氨酰顺反异构酶活性, 从而抑制 $\mathrm{T}$ 淋巴细胞特 异性转录因子(NK-AT)的活化及白细胞介素-2 等细胞因 子的合成，达到免疫抑制的药效. 他克莫司具有免疫抑 制药效强、用药剂量低、急性排异反应发生率低等优点,
在器官移植和自身免疫系统疾病方面疗效显著 ${ }^{[2]}$. 他克 莫司在临床上常用作肾脏、肝脏、胰腺等实体器官移植 手术后的免疫抑制剂，也可用于类风湿性关节炎、狼疮 性肾炎、特异性皮炎等自身免疫性疾病的治疗.

他克莫司 ${ }^{[1 a]}$ 与子囊霉素(Ascomycin, FK520) ${ }^{[3]}$ 、雷帕 霉素(Rapamycin) ${ }^{[4]}$ 在化学结构上具有很多共同点, 属于 天然的结构类似物(图 1): 三者的聚酮骨架环都包含相 同的哌啶单元，都有一个侧链包含 4-羟基-3-甲氧基环 己基结构；而他克莫司与子囊霉素的相似度更高，在相

\footnotetext{
*E-mail: znxu@zju.edu.cn

Received December 5, 2012; revised January 6, 2013; published online January 11, 2013

Project supported by the National Natural Science Foundation of China (Nos. 20832009, 21176214, 91213303), the National Basic Research Program of China (973 Program, Nos. 2010CB833200, 2012CB721100).

国家自然科学基金(Nos. 20832009, 21176214, 91213303)、国家重点基础研究发展计划(973 计划, Nos. 2010CB833200, 2012CB721100).
} 


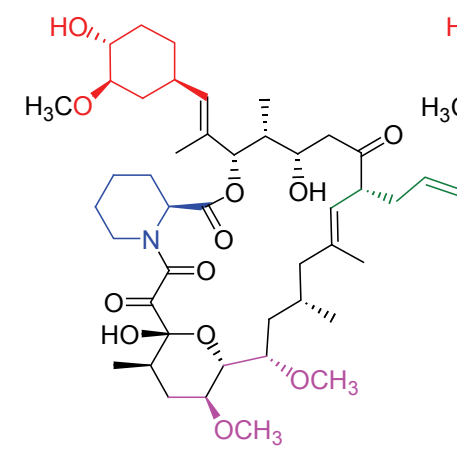

Tacrolimus, FK506

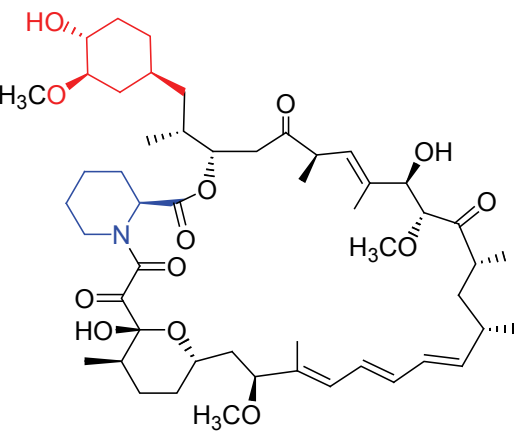

Rapamycin

图 1 他克莫司、子囊霉素和雷帕需素的化学结构式

Figure 1 Chemical structures of tacrolimus, ascomycin and rapamycin

同位置都包含两个甲氧基侧链. 两者的唯一差别在于他 克莫司含有一个罕见的烯丙基侧链, 而子囊霉素在相应 位置是乙基官能团.

他克莫司、子囊霉素和雷帕霉素都是来源于土壤链 需菌的大环内酯类天然产物, 而编码这类次级代谢产物 生物合成的相关基因在链需菌基因组中往往成族存在. 1995 年, Leadlay 课题组 ${ }^{[5]}$ 首次报道了 Streptomyces hygroscopicus NRRL5491 中的雷帕霉素基因簇 ${ }^{[5]} ; 1997$ 年, Motamedi 等 ${ }^{[6]}$ 报道了 Streptomyces sp. MA6548 中的部 分他克莫司基因簇; 2000 年, Reeves 课题组 ${ }^{[7]}$ 首次报道 了 Streptomyces hygroscopicus var. ascomyceticus ATCC14891 中的子囊霉素基因族; 2011 年, Yoon 课题组 ${ }^{[8]}$ 报 道了三株链霉菌 Streptomyces sp. KCTC11604BP, Streptomyces sp. ATCC55098(MA6858)和 Streptomyces kanamyceticus KCTC9225 中的完整的他克莫司基因族. 这些基因簇信息的获得极大地推进了他克莫司、子囊霉 素和雷帕霉素生物合成机制的研究.

\section{1 他克莫司骨架结构的形成机制}

他克莫司与子囊需素、雷帕需素的生物合成基因簇 都能编码典型的 I 型聚酮合酶(PKS)-非核糖体肽合成酶 (NRPS)杂合系统 ${ }^{[9]}$, 负责催化骨架内酯环结构的形成.
以他克莫司为例, 其骨架结构是由 3 个 I 型 PKS (FkbA, $\mathrm{FkbB}, \mathrm{FkbC}$ )和 1 个 NRPS (FkbP)催化形成的一个含有酰 胺键的二十三元大环内酯(图 2) ${ }^{[6 a]}$.

I 型 PKS 和 NRPS 都是以模块形式存在的多功能酶. 每一模块含有一套独特的、非重复使用的催化功能域, 负责完成一轮链延伸反应. 由 I 型 PKS 参与合成的天然 产物主要是聚酮类化合物, 例如红需素、阿维菌素等 ${ }^{[10]}$. 其延伸模块含有 $\beta$-酮基硫酯合成酶(KS)、酰基转移酶 (AT)和酰基载体蛋白(ACP)三个基本功能域，可能还包 含 $\beta$-酮基还原酶(KR)、脱水酶( $\mathrm{DH})$ 和烯醇还原酶(ER) 功能域. 起始模块(Loading module)负责识别起始单元, 将其上载至 ACP 形成硫酯. 延伸模块中的 AT 识别不同 的延伸单元(以丙二酰辅酶 $\mathrm{A}$ 和甲基丙二酰辅酶 $\mathrm{A}$ 最为 常见), 并将其转移至同模块的 ACP 上. 各延伸模块的 $\mathrm{KS}$ 将上游的聚酮链转移至自身的半胱氨酸活性位点上, 并催化同模块中 ACP 上的硫酯脱去二氧化碳形成碳负 离子, 进而与半胱氨酸残基上的聚酮链上的酰基发生 Claisen 缩合, 以完成多轮两碳单位的延伸 ${ }^{[11]}$. NRPS 主 要参与以万古霉素、环孢菌素 $\mathrm{A}^{[12]}$ 为代表的聚肽类天然 产物的生物合成. 其延伸模块包括至少三个催化功能 域: 缩合 $(\mathrm{C})$ 结构域、腺苷化 $(\mathrm{A})$ 结构域、肽酰转运蛋白 (PCP). A 功能域负责底物的选择和活化, 并将其转移至 PCP 功能域上形成氨酰化硫酯, C 功能域催化 PCP 上氨 (肽)酰化硫酯的氨基与上游模块中的硫酯缩合形成肽 键 ${ }^{[13]}$.

在他克莫司基因簇中, 编码 3 个 PKS 和 1 个 NPRS 的基因距离紧密. Motamedi 等 ${ }^{[6 b]}$ 最早指出, 他克莫司骨 架的聚䣶长链部分是以 FkbB-FkbC-FkbA 的催化顺序获 得的. 作为典型的 $\mathrm{I}$ 型 $\mathrm{PKS}, \mathrm{FkbB} 、 \mathrm{FkbC}$ 和 $\mathrm{FkbA}$ 分别 含有 5 个、 2 个和 4 个模块. 自 FkbB 的起始模块识别和 上载第 1 个前体开始, 以硫酯键连接在 $\mathrm{ACP}$ 上的聚酤链 连续发生 10 轮 Claisen 缩合反应, 所获得的全长的线性 聚酩链连接于 $\mathrm{FkbA}$ 最后一个模块的 ACP 上. 作为骨架 结构组装体系中唯一的 NRPS, FkbP 负责催化骨架组装 的最后步骤. Walsh 课题组 ${ }^{[14]}$ 揭示了 FkbP 仅有的模块所 含有的特殊 $\mathrm{C}_{1}-\mathrm{A}-\mathrm{PCP}-\mathrm{C}_{2}$ 功能域. 其中, $\mathrm{C}_{1}-\mathrm{A}-\mathrm{PCP}$ 功能 域能够以特殊的 N7-C6 酰胺键向他克莫司的骨架引入 1 个氨基酸. 同时, $\mathrm{C}_{2}$ 功能域被认为可以在 $\mathrm{C}(1)-\mathrm{C}(26)$ 处 形成 $\mathrm{C}-\mathrm{O}$ 内酯键, 从而催化大环内酯骨架的形成.

\section{2 途径特异性前体的生物合成机制}

负责组装他克莫司骨架的杂合 PKS/NRPS 系统总 共能够识别和上载 12 分子的前体, 除了常见的丙二酰 辅酶 $\mathrm{A}$ (2 分子)和甲基丙二酰辅酶 $\mathrm{A}$ (5 分子)外, 还包括 1 分子的 4,5-二羟基-1-烯-环已酸(DHCHC), 2 分子的甲氧 


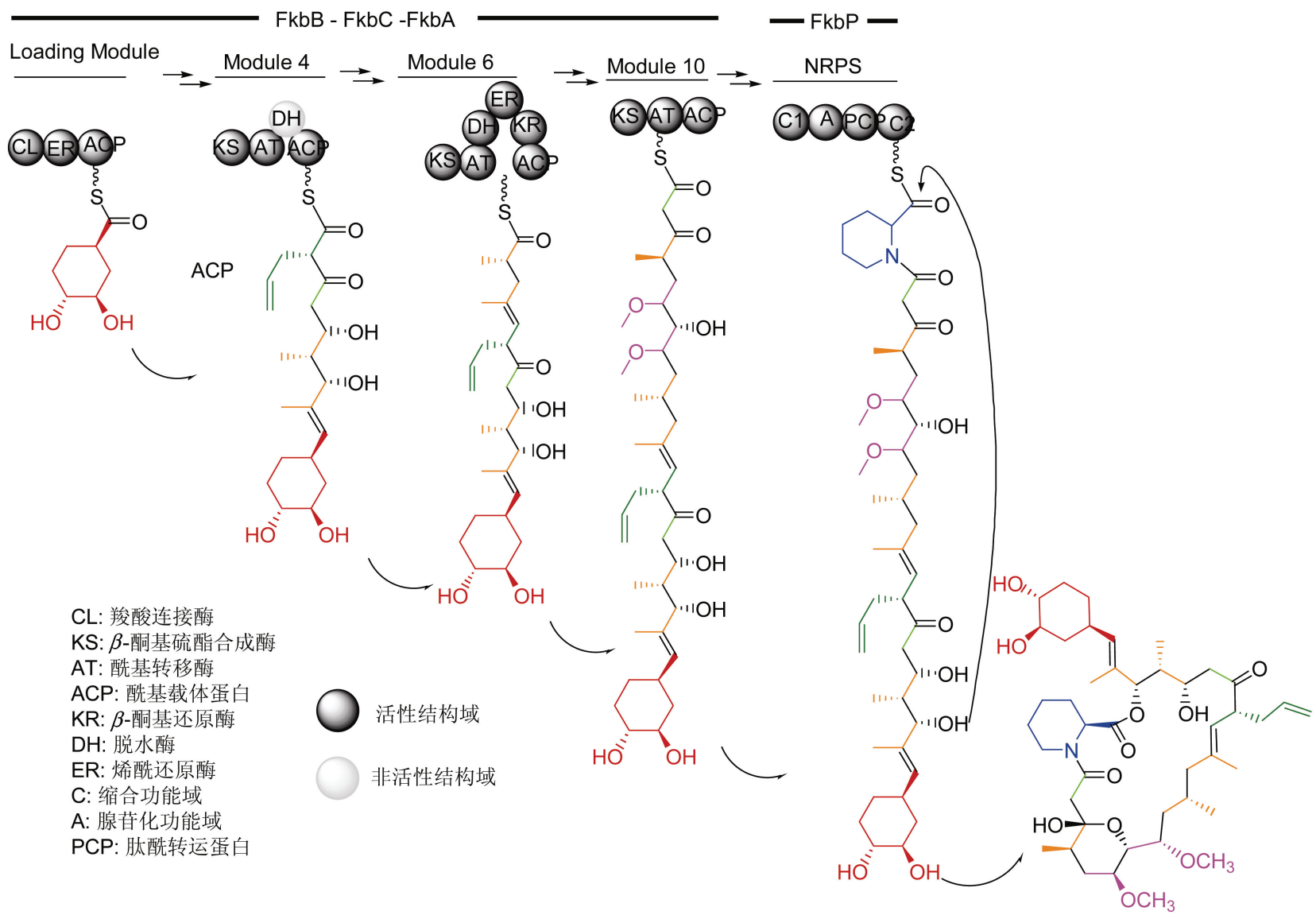

图 2 他克莫司骨架结构的形成机制

Figure 2 Generation of the macrolide skeleton of tacrolimus

基丙二酰 ACP, 1 分子的烯丙基丙二酰辅酶 $\mathrm{A}$ 和 1 分子 的哌啶甲酸 ${ }^{[15]}$. 这些特殊前体的引入是致使他克莫司 具备独特化学结构和药理活性的最大原因. 值得一提的 是，这些特殊的 PKS/NPRS 前体并非直接来源于初级代 谢. 随着他克莫司生物合成基因簇的报道以及随后对其 中各个基因功能的研究, 人们发现编码这 4 种前体的生 物合成的相关基因全部位于他克莫司的基因簇中. 也就 是说，这 4 种被 PKS/NRPS 杂合系统识别的特殊前体具 有途径特异性(pathway-specific). 以下将分别介绍这些 途径特异性前体具体的生物合成机制.

\subsection{PKS 起始单元 4,5-二羟基-1-烯-环己酸( $\mathrm{DHCHC})$}

他克莫司以及子囊需素、雷帕霉素的侧链都包含有 特殊的 4-差基-3-甲氧基环己基结构. Paiva 等 ${ }^{[16]}$ 在向雷 帕霉素产生菌喂养 ${ }^{13} \mathrm{C}$ 标记的莽草酸后, 发现产物相应 位置获得标记. Reynolds 等 ${ }^{[17]}$ 用 ${ }^{13} \mathrm{C}$ 和 $\mathrm{D}$ 标记的莽草酸 喂养子囊霉素产生菌, 也得到了被标记的子囊霉素. 由 此推断, 他克莫司侧链上的相同六元环结构也应该来源 于谷草酸. 通过序列分析和比对, Lowden 等 ${ }^{[18]}$ 指出 PKS 起始模块含有 3 个特殊催化功能域, 分别为羧酸连接酶
(CL), ER 和 ACP. CL 功能域具有三磷酸腺苗(ATP)依赖 的连接酶活性, 能够催化形成单磷酸腺苷(AMP)活化的 羧酸, 继而将活化的羧酸转移到下游 $\mathrm{ACP}$ 上. 他们推测 他克莫司和子囊霉素、雷帕霉素的真实起始单元应该是 游离羧酸形式的. ER 功能域具有还原酶活性, 故上载到 $\mathrm{ACP}$ 的结构单元应该是不饱和的. Reynolds 等 ${ }^{[17]}$ 的标记 实验结果指出莽草酸 $\mathrm{C}(3)$ 位置的羟基和 $\mathrm{C}(6)$ 上的一个 氢没有转移到最终产物, 故莽草酸的不饱和六元环结构 可能先发生 1,4-消除反应脱水, 再通过 1,4-加成反应加 氢, 得到在 $C(1)-C(2)$ 处呈不饱和烯键的真实起始单元. 此外, Motamedi 等 ${ }^{[19]}$ 的研究指出, 4-羟基-3-甲氧基环己 基侧链上的甲氧基是通过后修饰作用在 $O$-甲基转移酶 的催化下得到的. 综合以上分析, Lowden 等 ${ }^{[20]}$ 提出，他 克莫司、子囊霉素和雷帕霉素的生物合成起始单元为 4,5-二差基-1-烯-环己酸(DHCHC). 至此，4-羞全基-3-甲氧 基环已基结构的前体已经被确证是 DHCHC (Scheme 1), 但是对 DHCHC 的生物合成机制却并不清楚.

通过基因功能的证实, DHCHC 的形成机制才最终 获得确立. Gregory 等 ${ }^{[21]}$ 通过基因敲除和回补实验，在雷 帕霉素的基因簇中找到了与 $\mathrm{DHCHC}$ 生物合成相关的基 


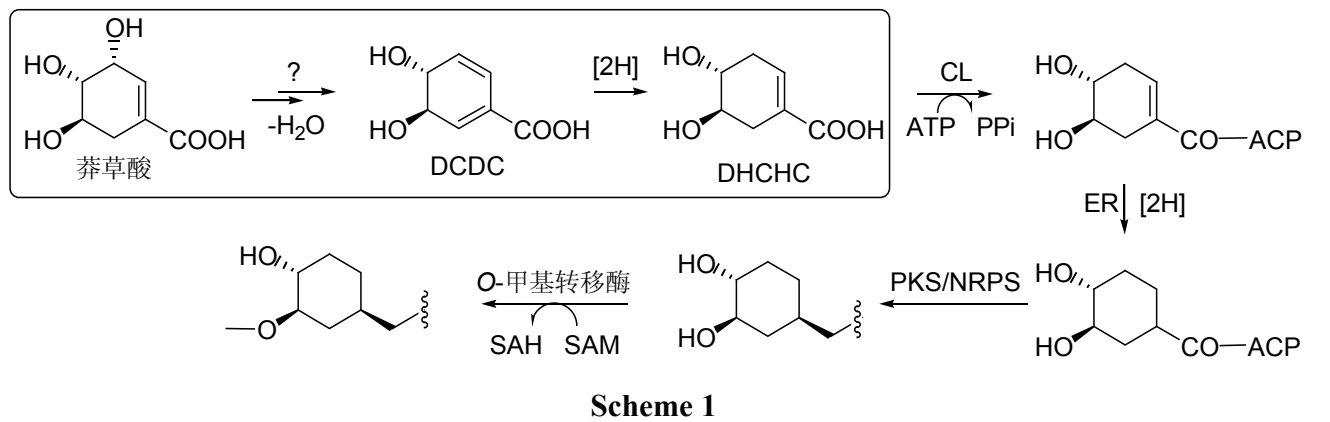

因 rapK. rapK 在他克莫司和子囊霉素基因簇中的同源 基因是 $f k b O$. RapK 和 $\mathrm{FkbO}$ 曾经被认为能够辅助骨架结 构 $\mathrm{C}(9)$ 位置的氧化反应, 参与酮基的形成 ${ }^{[22]}$. 为了确证 蛋白的催化活性, Andexer 等 ${ }^{[23]}$ 以莽草酸为底物分别对 RapK 和 FkbO 进行了体外测活, 结果都没有检测到产物 的生成. 由于蛋白序列分析结果提示 RapK 和 FkbO 与 分支酸变位酶具有很高的同源性, 他们尝试以分支酸为 底物的体外测活, 成功检测到了 3,4-二羟基-1,5-二烯-环 己酸(DCDC)和丙酮酸的产生. 由此证明, DCDC 的生物 合成是以分支酸为底物的水解反应，而不是先前推测的 以荓草酸为底物的 1,4-消除反应(Scheme 2). 这与标记 实验的结果并不相悖, 因为在初级代谢中, 分支酸是莽 草酸的下游代谢产物 ${ }^{[24]}$. 莽草酸顺次经过莽草酸激酶 以 ATP 为共底物的磷酸化、5-烯醇丙酩酰-䒭草酸-3-磷 酸(EPSP) 合酶以磷酸烯醇式丙酮酸为共底物的加成消 除和分支酸合酶催化的 1,4-反式消除 3 步反应 ${ }^{[25]}$, 转化 成为分支酸. RapK 和 FkbO 体外活性的检测从根本上阐 明了 $\mathrm{DHCHC}$ 的生物合成机制, 也拓宽了人们对分支酸 后续代谢途径多样性的认识.

\subsection{PKS 延伸单元甲氧基丙二酰 ACP}

雷帕霉素的骨架结构含有两个氧甲基侧链, Gregory 等 ${ }^{[26]}$ 的研究表明, 这两个氧甲基是通过 $O$-甲基转移 酶 RapQ 和 RapM 的后修饰获得的. 不同于雷帕霉素,
Byrne 等 ${ }^{[27]}$ 通过同位素标记实验证明他克莫司和子囊需 素骨架结构中的两个氧甲基并非来自乙酸单元，而是来 源于具有 “乙醇酸” 结构的葡萄糖或者甘油. 安丝菌素 (Ansamitocin)和子囊需素的骨架结构中都含有甲氧基侧 链. Floss 课题组 ${ }^{[28]}$ 对比两者的生物合成基因簇 ${ }^{[7,28 b, 29]}$, 发现安丝菌素基因簇中的 asm13 asm 17 与子囊霉素基 因簇中的 $f k b G \sim f k b K$ 具有极高的同源性. 基因序列分 析显示, $a s m 14$ 和 $f k b J$ 能够编码 $\mathrm{ACP}, a s m 17$ 和 $f k b G$ 则 能够编码 $O$-甲基转移酶. 他们大胆猜测, 由 5 个连锁基 因组成的亚基因簇能够催化形成前体甲氧基丙二酰 ACP.

Kato 等 ${ }^{[28 a]}$ 为此设计了异源表达实验，将携带有 asm 13 asm 17 基因的穿梭质粒 pHGF9251 导入到 Streptomyces lividans K4.114/pKOS38-187. pKOS38-187 是由 Reeves 等 ${ }^{[30]}$ 构建的，携带有改造的 eryA 基因. 原 始的 EryA 能够催化 6-脱氧红霉内酯 B(6-DEB)的合成, 但由于其中第 6 个模块的 $\mathrm{AT}$ 被来自 $\mathrm{FkbA}$ 的 AT8 替换, 6-DEB 的 C2 位置会因为引入不同的 PKS 延伸单元而发 生变化. 向异源表达宿主 S. lividans K4.114 导入 pKOS38-187 后，能够检测到 2-脱甲基-6-DEB (1.5 $\mathrm{mg} / \mathrm{L})$ 和 6-DEB $(0.5 \mathrm{mg} / \mathrm{L})$ 的生成. 而将 $\mathrm{pHGF} 9251$ 和 pKOS38-187 共同导入 S. lividans K4.114 后, 则只检测 到 2-脱甲基-2-甲氧基-6-DEB $(3 \mathrm{mg} / \mathrm{L})$ 的产生，而没有 2脱甲基-2-羟基-6-DEB(图 3). Kato 等 ${ }^{[28]}$ 认为, 之所以产<smiles></smiles><smiles>O=CC1=CC[C@@H](O)[C@H](O)C1</smiles><smiles>O=C(O)C1=C[C@H](OC[18OH])[C@@H](O)[C@H](O)C1</smiles><smiles>C=C(OCCCCO)C(=O)O</smiles><smiles>C=C(O[C@H]1CC(C(=O)O)=C[C@H](OC[P+]#P)[C@@H]1O)C(=O)O</smiles><smiles>O=C(O)C1=CC(O)[C@H](O)C=C1</smiles>

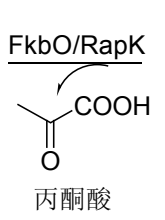<smiles>C=C(C)O[C@@H]1C=C(C(=O)O)C[C@H]1O</smiles>

Scheme 2 


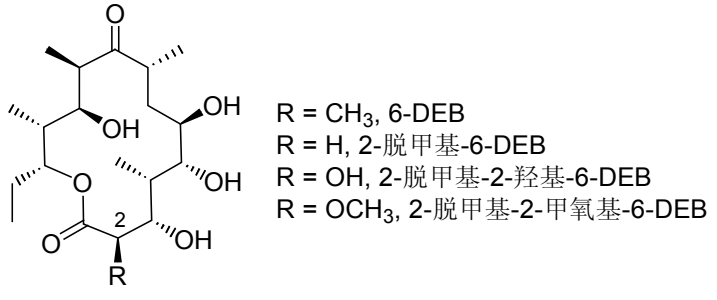

图 3 6-脱氧红霉内酯 B 系列化合物的化学结构式 Figure 3 Chemical structures of the 6-deoxyerythronide series

生这种现象是因为来自 $\mathrm{FkbA}$ 的 AT8 特异地上载由 Asm13 Asm17 (或同源蛋白 FkbG FkbK) 编码的 2-甲 氧基丙酸单元, 或者在上载 2-羟基丙酸单元后进一步发 生甲基化修饰. 至此, Asm17 和 $\mathrm{FkbG}$ 作为 $O$-甲基转移 酶的功能已经获得确证, 但其发挥活性是在延伸单元上 载前还是上载后仍不得而知. 也就是说, PKS 延伸单元 究竟是甲氧基丙二酰 ACP 还是羟基丙二酰 ACP 尚不明 确. 为此, Kato 等 ${ }^{[28 a]}$ 进一步构建了敲除 asm17 的 pHGF9251 衍生质粒 pHGF9263, 并将其与 pKOS38-187 共转化到 $S$. lividans K4.114. 产物检测结果与单独导入 pKOS38-187 的情况类似, 没有 2-脱甲基-2-甲氧基 -6-DEB 或者 2-脱甲基-2-羟基-6-DEB. 由此可见, Asm17 和 FkbG 是在延伸单元上载前起作用的, PKS 识别的延 伸单元是甲氧基丙二酰 ACP, 而非羟基丙二酰 ACP.

基因序列分析 $a s m 13 \sim a s m 17$ 和 $f k b G \sim f k b K$ 亚基因 族中的另 3 对同源基因, $a s m 13$ 和 $f k b K$ 负责编码 $\beta$-羟基 酰基辅酶 $\mathrm{A}$ 脱氢酶, asm 15 和 $f k b I$ 负责编码酰基辅酶 $\mathrm{A}$ 脱氢酶, 而 $a s m 16$ 和 $f k b H$ 则编码卤酸脱卤酶 $(\mathrm{HAD})^{[31]}$. Watanabe 等 ${ }^{[32]}$ 通过蛋白结晶和结构解析, 指出 FkbI 识 别的底物更可能是酰化 ACP, 而非酰化辅酶 A. 换言之, FkbI 和 Asm15 应该是一种酰基 ACP 脱氢酶, FkbK 和 Asm13 也就有可能是 $\beta$-羟基酰基 ACP 脱氢酶. Kato 等 ${ }^{[28 a]}$ 提出, Soraphen 的生物合成基因簇也含有类似的亚基 因簇, 并且除 $a s m 16$ 和 $f k b H$ 以外的其它 4 对基因都能在 其中找到同源基因或者功能域. 由于 Soraphen 亚基因簇 中的 $\operatorname{sor} C$ 所额外编码的 $\mathrm{AT}$ 和 $\mathrm{ACP}$ 两个功能域被认为 参与底物氨基酸的活化和修饰 ${ }^{[33]}$, 他们推测 Asm16 和 $\mathrm{FkbH}$ 也发挥类似的功能. Dorrestein 等 ${ }^{\left[{ }^{[1]}\right.}$ 以噁唑霉素 (Oxazolomycin)生物合成基因簇中 $\mathrm{FkbH}$ 的同源蛋白 OzmB 为研究对象, 检测到了甘油酰转移酶和磷酸酶的 双功能, 并证明其作用底物为 1,3 -二磷酸甘油酸 (1,3-BPG). Chan 等 ${ }^{[34]}$ 总结前人工作, 推导了由 $\mathrm{FkbG〜}$ FkbK 催化的 PKS 延伸单元甲氧基丙二酰 ACP 的生物合 成机制(Scheme 3): 首先, FkbH 结合糖酵解途径中间体 1,3-BPG，使之脱磷后以硫酯键靶定到 ACP 即 FkbJ 上; 随后, FkbK 催化甘油酰 ACP 的氧化, 形成 2-羟基-3-氧 代丙酰 ACP; FkbI 进一步氧化 FkbK 的产物得到羟基丙

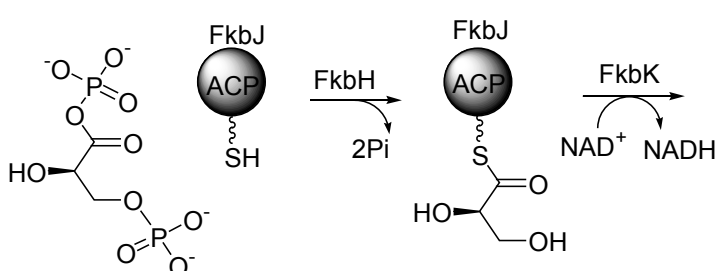

1,3-BPG
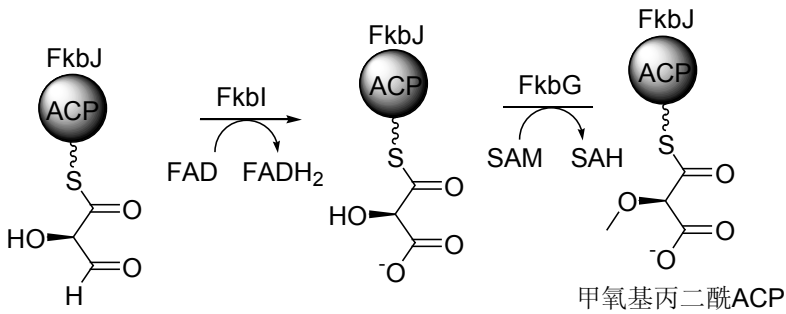

\section{Scheme 3}

二酰 $\mathrm{ACP}$; 最后, $\mathrm{FkbG}$ 完成羟基位置的 $O$-甲基化得到 甲氧基丙二酰 ACP. 含有甲氧基侧链的聚酮化合物不 胜枚举，甲氧基丙二酰 ACP 生物合成途径的揭示将有 助于其它具有类似结构的活性天然产物的研究.

\subsection{PKS 延伸单元烯丙基丙二酰辅酶 $\mathrm{A}$}

他克莫司 $C(21)$ 上的烯丙基侧链十分特殊，也是其 与子囊霉素唯一的结构差别所在. Paiva 等 ${ }^{[35]}$ 通过 ${ }^{13} \mathrm{C}$ 标 记实验证明引入烯丙基单元的前体包含 1 分子丙酸和 1 分子乙酸，提示这种前体很可能是通过类似 PKS 机制 形成的五碳单元. 不同于子囊霉素和雷帕霉素, 他克莫 司的基因簇在过去很长一段时间内没有获得完整报道， 已知序列并不包含与五碳单元前体相关的基因. 因此, 五碳单元前体的具体结构及其生物合成机制一直没有 得到确立. 直至近年, Petkovic 课题组 ${ }^{[36]}$ 和 Yoon 课题组 ${ }^{[8]}$ 分别报道了位于 $f k b G \sim f k b K$ 亚基因簇上游的另一组 $t c s$ (或命名为 $a l l$ )亚基因簇，对引入烯丙基侧链结构的 PKS 延伸单元的研究才取得突破性进展.

通过基因簇差异分析, Petkovic 课题组 ${ }^{[36]}$ 和 Yoon 课 题组 ${ }^{[8]}$ 在子囊霉素 $f k b G \sim f k b K$ 亚基因簇上游负责合成 乙基丙二酰辅酶 $\mathrm{A}$ 的相应位置发现一组截然不同的基 因. Yoon 课题组 ${ }^{[8]}$ 还比对了 3 株产生菌的他克莫司基因 簇, 指出只有 $t c s A \sim t c s D(a l l A \sim$ allD $) 4$ 个基因是五碳单 元前体生物合成所必需的，这一点也被基因敲除实验证 明. 从蛋白序列角度看, TcsA(AllA)含有 AT-ACP 双功 能域, TcsB (AllK)含有 KS 功能域, 两者很可能协同完成 特殊聚酮前体的第一步缩合反应; $\mathrm{T} \operatorname{csC}(\mathrm{AllR})$ 与巴豆酰 辅酶 $\mathrm{A}$ 羧化/还原酶(CCR) 同源, $\mathrm{TcsD}(\mathrm{AllD})$ 与 FkbI 类似, 属于酰基 ACP 脱氢酶, 推测参与羧化、还原、脱氢修饰 以最终形成引入他克莫司骨架的前体. Petkovic 课题组 ${ }^{[36]}$ 采用同框敲除的方法构建了 allK 基因失活的突变株. 在不影响周边基因的情况下, 突变株发酵产物中的他克 
莫司被完全中断，而副产品子囊霉素的产量基本没有变 化. 这就说明 all 亚基因簇是与他克莫司的生物合成直 接相关的, 并且极可能负责合成他克莫司中与子囊霉素 延伸单元乙基丙二酰辅酶 $\mathrm{A}$ 平行的前体. 他们化学合成 了与酰基 $-N$-乙酰半胱氨酸硫酯(SNAC) 以硫酯键连接的 聚酮前体(图 4), 向 allK 同框敲除突变株喂养烯丙基丙 二酰 SNAC, 在发酵产物中检测到了他克莫司. 由此证 明, 由 all 亚基因簇催化形成的 PKS 延伸单元极可能是 烯丙基丙二酰辅酶 A. Yoon 课题组 ${ }^{[8]}$ 的研究采取类似策 略, 分别构建了 $t \operatorname{cs} A, t \operatorname{cs} B, t \operatorname{cs} C$ 和 $t c s D$ 同框敲除突变株, 发酵产物的检测显示他克莫司的生物合成都被中断了. 向 $\operatorname{tcs} A$ 同框敲除突变株喂养 3-氧代戊酰 SNAC 或者反 式 2-戊烯酰 SNAC 都无法恢复他克莫司的产生, 这种情 况对 $t c s B$ 同框敲除突变株则不然. TcsA 具有 $\mathrm{AT}$ 和 ACP 双功能域, 可以利用 $\mathrm{AT}$ 活性将 SNAC 上聚酮链转移到 自身的 ACP. 这就是说, 由 $\mathrm{T} \operatorname{cs} \mathrm{A} \sim \mathrm{T} \operatorname{csD}$ 催化的生物合 成途径的中间体应该是以硫酯键连接在 $\mathrm{ACP}$ 上的聚酮 链. 换言之, $\mathrm{FAS}$ 和 $\mathrm{TesC}$ 的作用底物分别是 3 -氧代戊酰 $\mathrm{ACP}$ 和反式 2-戊烯酰 ACP. 向 $t c s A$ 和 $t c s B$ 同框敲除突 变株喂养丙基丙二酰 SNAC 都无法恢复他克莫司的产 生, 说明丙基丙二酰辅酶 $\mathrm{A}$ 可能无法上载到 TcsA 的 ACP 功能域, 也提示 TcsD 的作用底物应该是丙基丙二 酰 ACP. 向 $\operatorname{tsc} C$ 同框敲除突变株喂养 3-氧代戊酰 SNAC 和反式 2-戊烯酰 SNAC 都无法恢复他克莫司的产生, 说 明 $\mathrm{TcsC}$ 的还原羧化活性对烯丙基丙二酰辅酶 $\mathrm{A}$ 的生物 合成而言必不可少. $t c s D$ 同框敲除突变株的发酵产物累 积有大量 $C(36)-C(37)$ 位置饱和的他克莫司类似物, 向 其喂养除烯丙基丙二酰 SNAC 外的任何 SNAC 替代物 都无法对发酵产物产生显著影响, 说明 TcsD 应该在 $\mathrm{T} \operatorname{cs} \mathrm{A} \sim \mathrm{T} \operatorname{csC}$ 之后的最后步骤发挥催化活性的. 由于 $\mathrm{TcsD}$ 的活性在 $\operatorname{tcs} B$ 和 $t c s C$ 同框敲除突变株中不受影响, $\mathrm{C}(36)-\mathrm{C}(37)$ 位置饱和的他克莫司类似物的出现提示 $\mathrm{TcsD}$ 是在延伸单元上载到 PKS 前催化形成不饱和烯键的.<smiles>C=CCC(C(=O)O)C(=O)SCCNC(C)=O</smiles>
烯丙基丙二酰SNAC<smiles>CC/C=C/C(=O)SCCNC(C)=O</smiles>
2-戊烯酰SNAC 3-氧代戊酰SNAC<smiles>CCCC(C(=O)O)C(=O)SCCNC(C)=O</smiles><smiles>CCC(=O)CC(=O)SCCNC(C)=O</smiles>

丙基丙二酰SNAC

图 4 化学喂养的 SNAC 聚酮底物

Figure 4 Polyketide Substrates linked to SNAC for chemical complementation

基于大量实验事实, Yoon 课题组 ${ }^{[8]}$ 推导出 PKS 延伸
单元烯丙基丙二酰辅酶 $\mathrm{A}$ 的生物合成机制. TcsA(AllA) 的 AT-ACP 双功能域和 TcsB(AllK)的 KS 功能域组成“最 小 PKS”, 共同催化 1 分子丙酰辅酶 $\mathrm{A}$ 起始单元和 1 分 子丙二酰辅酶 A 延伸单元发生 Claisen 缩合, 形成靶定 在 ACP 上的 3-氧代戊酸结构. 初始形成的聚酩链经过 宿主脂肪酸合成酶(FAS)催化的 $\beta$-酮基还原和脱水、 $\mathrm{T} \operatorname{csC}(\mathrm{AllR})$ 的还原和羧化修饰以及 $\mathrm{T} \operatorname{csD}(\mathrm{AllD})$ 的脱氢作 用得到烯丙基丙二酸结构单元. 烯丙基丙二酸单元极可 能通过未知酶的作用从 $\mathrm{ACP}$ 转移到辅酶 $\mathrm{A}$, 成为独一无 二的 PKS 延伸单元(Scheme 4).

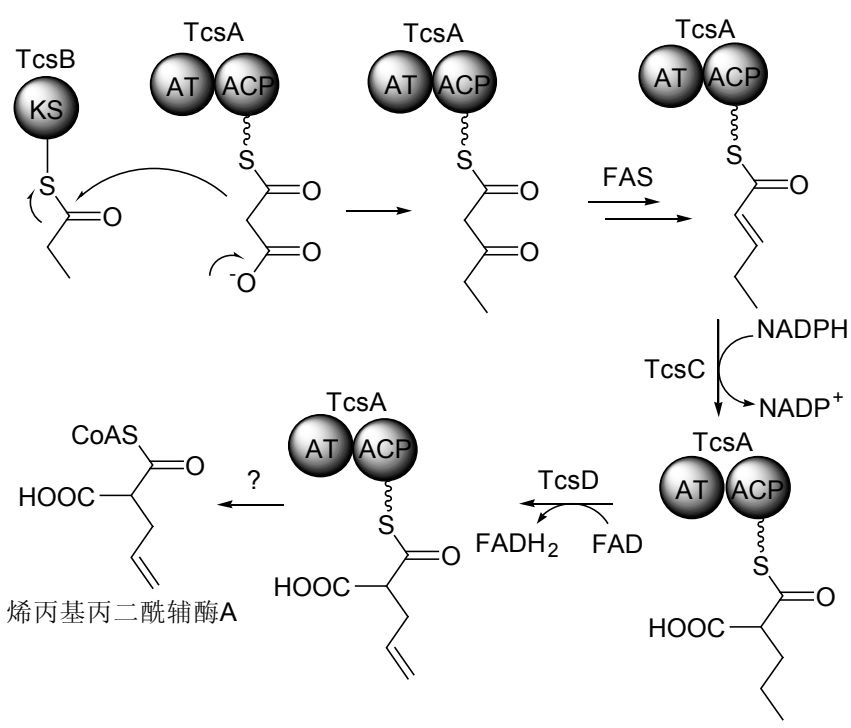

Scheme 4

\subsection{NRPS 延伸单元 $L-$-哌啶甲酸}

大环骨架上的五元哌啶环是他克莫司、子囊需素和 雷帕霉素共有的另一特殊结构. Walsh 课题组 ${ }^{[14]}$ 指出, 哌啶结构是由 PKS/NRPS 杂合体系中的 RapP(FkbP)引 入 1 分子非天然氨基酸形成的. Paiva 等 ${ }^{\left[{ }^{37]}\right.}$ 在 $L$-赖氨酸营 养缺陷条件下, 分别向雷帕霉素产生菌喂养 ${ }^{13} \mathrm{C}$ 标记的 $L$-赖氨酸和 ${ }^{3} \mathrm{H}$ 标记的 $D, L$-甲基哌啶, 发酵产物在哌啶 环的相应位置都获得同位素标记，且 $D, L$-甲基哌啶的 ${ }^{3} \mathrm{H}$ 同位素喂养获得很高的标记比例. 由此可见，负责引 入哌啶结构的 NRPS 延伸单元极可能是来源于 $L$-赖氨酸 的哌啶甲酸.

Leadlay 课题组 ${ }^{[5]}$ 通过分析雷帕霉素的生物合成基 因簇指出, rapL 负责编码环化脱氨酶, 很可能负责催化 $L$-赖氨酸直接转变成 $L$-哌啶甲酸. $r a p L$ 在他克莫司和子 囊霉素基因簇中的同源基因是 $f k b L$. Khaw 等 ${ }^{[38]}$ 构建了 敲除 rapL 的突变株, 发现雷帕霉素的生物合成被完全 中断，而向该突变株化学回补 $L$-甲基哌啶则能够恢复雷 帕霉素的产生，由此证明 RapL(FkbL)直接参与 NRPS 前 体 $L$-哌啶甲酸的生物合成. Walsh 课题组 ${ }^{[39]}$ 报道了 RapL 
在体外的赖氨酸环化酶活性, 从根本上解决了 $L$-哌啶甲 酸的生物合成机制问题. 他们结合催化活性已有报道的 鸟氨酸环化脱氨酶 $(\mathrm{OCD})$, 以 $L$-赖氨酸为底物, 添加烟 酰胺作为辅因子, 成功检测到了 $L$-哌啶甲酸的生成. 通 过测定 RapL 的体外反应动力学数据, 他们进一步提出 了 RapL 作为 $L$-赖氨酸环化脱氨酶的酶促反应机理是一 种 “复合物 $\mathrm{NAD}^{+}$依赖的转化” (Scheme 5). 首先, 氧化 态的烟酰胺 $\left(\mathrm{NAD}^{+}\right)$与 $\mathrm{RapL}$ 蛋白紧密结合, 辅助底物 $L-$ 赖氨酸的氧化, 自身转变成还原态 (NADH). 随后, 新生 的亚氨基酸在 RapL 蛋白的活性位点实现分子内的成环 和脱氨. 最后, NADH 作为氢供体辅助环化亚胺的还原, 所得产物 $L$-哌啶甲酸可以从蛋白上轻易地解离下来, 而 循环再生的 $\mathrm{NAD}^{+}$则继续与蛋白结合参与下轮的催化反 应.

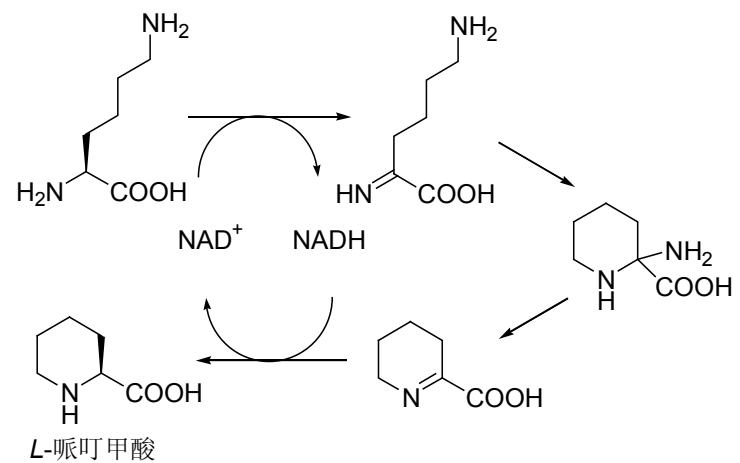

Scheme 5

\section{3 他克莫司骨架环后修饰}

在二十三元聚酮骨架形成之后, 还需要两步后修饰 最终得到成熟的他克莫司产物: $\mathrm{C}(9)$ 的酮基化和 $\mathrm{C}(31)$ 的 $O$-甲基化 ${ }^{[19 a]}$. Shafiee 等 ${ }^{[19 b, 40]}$ 通过基因敲除获得了他 克莫司骨架环形成后的中间体 9-脱酮基,31-脱甲基他克 莫司和 31-脱甲基他克莫司. 他们以 31-脱甲基他克莫司 为底物, 在测活体系中添加 $S$-腺苷甲硫氨酸 $(\mathrm{SAM})$ 作为 辅因子, 成功检测到 FkbM 的氧甲基化活性. 为了弄清 $\mathrm{C}(9)$ 酮基形成的酶学机制, 明确他克莫司骨架环后修饰 的具体路径, 我们针对 FkbD 和 FkbM进行了研究. 在体 内利用同框敲除的方法, 获得单独或者同时敲除 $f k b D$ 和 $f k b M$ 的突变株, 发酵产物的检测结果提示他克莫司 的生物合成后修饰酶 FkbD 和 FkbM 没有严格的作用顺 序(Scheme 6). 这一实验结果通过体外蛋白的催化活性 及相关酶促反应动力学数据的测定得到了确证. 我们还 在体外成功检测到了由细胞色素 P450 氧化酶 FkbD 负 责的连续氧化反应，即催化亚甲基发生羟化继而形成酮 基, 证实了 FkbD 能够独立催化他克莫司 $\mathrm{C}(9)$ 位 $\alpha$-酮基 的形成.

比较未成熟中间体与他克莫司的抗真菌活性, 可以
发现 9-脱酮基和 31- $O$-脱甲基的中间体的抗真菌活性均 有不同程度的降低. 正如大多数具有药理、生理活性的 天然产物，他克莫司的骨架环后修饰被证明对其生理活 性的贡献巨大.

\section{4 总结与展望}

他克莫司作为一种具有独特化学结构和重要药理 活性的大环内酯类化合物, 其化学全合成的研究引起了 大化学合成工作者的广泛兴趣, 这方面研究报道主要集 中在他克莫司大规模发酵生产得以实现之前. 由于他克 莫司分子量大、手性复杂、结构复杂，现有方法多采用 汇聚式合成策略 ${ }^{[4]}$. Merck 公司的研究人员 ${ }^{[42]}$ 于 1989 年 首次全合成获得他克莫司，这也是国际上首例三羰基大 环内酯类天然产物的全合成报道. 他们采用不对称 Evans-Aldol 缩合反应分别合成关键性的片段结构, 以 41 步 0.067\%的总产率得到终产物. 1990 年, Schreiber 课 题组 ${ }^{[43]}$ 采用与 Merck 公司相似的合成策略并采用 Ireland-Claisen 重排等新颖的方法，通过更高效率地获 得甲基哌啶单元，最终以 32 步 $0.21 \%$ 的总产率合成了他 克莫司. 随后, Ireland 课题组 ${ }^{[44]} 、 S i h$ 课题组 ${ }^{[45]}$ 、 Danishefsky 课题组 ${ }^{[46]}$ 、Smith 等 ${ }^{[47]}$ 通过发展新的合成方 法、改进部分结构单元的合成路径, 丰富和完善了他克 莫司的化学合成研究.

时至今日, 关于他克莫司生物合成机制的研究已经 十分全面、彻底. 而其生物合成机制的揭示, 特别是途 径特异性前体形成机制的阐明，使得科研人员的目光更 多的聚焦到通过基因工程改造的方法获得产量提高、组 分优化的新一代他克莫司产生菌上来. 例如, 我们 ${ }^{[15 a]}$ 利用位点特异性整合的方法，在他克莫司产生菌的基因 组中倍增了与前体甲氧基丙二酰 ACP 和烯丙基丙二酰 辅酶 $\mathrm{A}$ 生物合成相关的亚基因簇, 获得的基因重组菌的 他克莫司产量得到约 $120 \%$ 的提高. Kosec 等 ${ }^{[48]}$ 发现 $\mathrm{AllR}(\mathrm{Tcs} C)$ 的 CCR 活性也会参与乙基丙二酰辅酶 $\mathrm{A}$ 的 生物合成而导致发酵成品中出现子囊霉素副产物. 他们 向敲除 allR 基因的突变株喂养烯丙基丙二酰 SNAC, 获 得的发酵成品中他克莫司的组分比例大大提高.

除此之外，特殊前体生物合成机制的确定为人们获 得新型他克莫司结构类似物提供了充分的依据. Moore 课题组 ${ }^{[49]}$ 改造了特殊前体烯丙基丙二酰辅酶 $\mathrm{A}$ 的体内 合成途经，利用海洋链霉菌来源的 Ansalactam A 基因簇 中负责编码异丁基丙二酰-ACP 生物合成的 3 个基因 $a n l E \sim a n l G$ 代替他克莫司原本负责编码丙基丙二酰$\mathrm{ACP}$ 生物合成的 $\operatorname{tcs} A \sim \operatorname{tcs} C$ (或名 $a l l A \sim a n l R$ ), 继而在 $t c s D$ (或名 allD)的脱氢作用下形成异丁酰单元，并最后 由 PKS 引入到他克莫司的聚酮结构, 形成 C(36)甲基化 


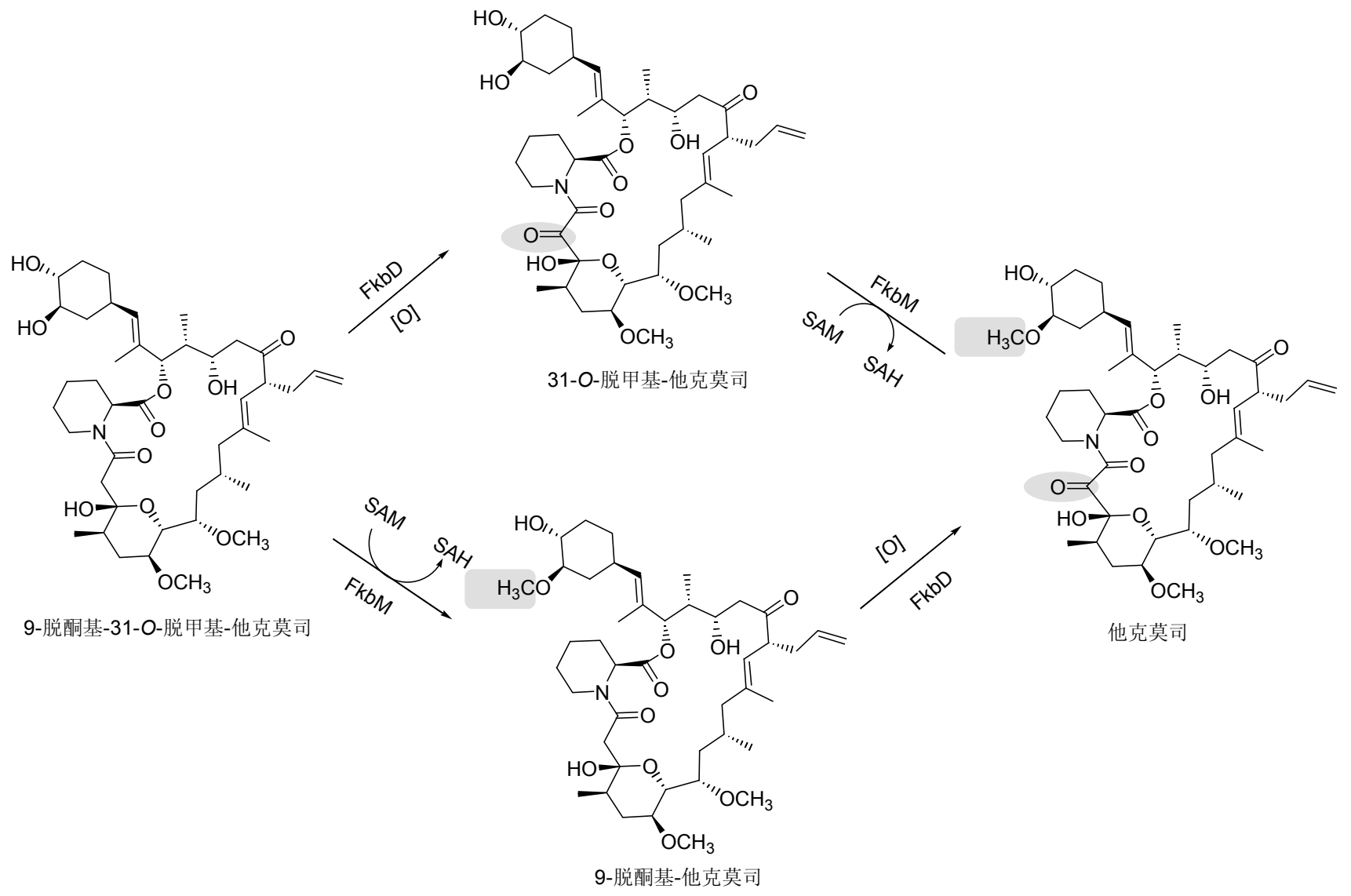

Scheme 6

的他克莫司结构类似物. 与此同时, 突变合成和化学互 补的方法也具有很大的开发潜力. 这样的例子在雷帕霉 素的结构改造尝试中已有多例成功范本. 例如, Lowden 等 ${ }^{[50]}$ 向敲除 $r a p K$ 的突变株喂养 DHCHC 类似物, Khaw 等 ${ }^{[38]}$ 向敲除 $r a p L$ 的突变株喂养 $L$-哌啶甲酸类似物, 都 获得了相应位置发生变化的雷帕霉素结构类似物. 同样 的方法和策略完全可以应用到他克莫司结构的改造中.

\section{References}

[1] (a) Kino, T.; Hatanaka, H.; Hashimoto, M.; Nishiyama, M.; Goto, T.; Okuhara, M.; Kohsaka, M.; Aoki, H.; Imanaka, H. J. Antibiot. 1987, 40, 1249.

(b) Kino, T.; Hatanaka, H.; Miyata, S.; Inamura, N.; Nishiyama, M.; Yajima, T.; Goto, T.; Okuhara, M.; Kohsaka, M.; Aoki, H.; Ochiai, T. J. Antibiot. 1987, 40, 1256.

[2] Liu, J.; Farmer, J. D.; Lane, W. S.; Friedman, J.; Weissman, I.; Schreiber, S. L. Cell 1991, 66, 807.

[3] Hatanaka, H.; Kino, T.; Miyata, S.; Inamura, N.; Kuroda, A.; Goto, T.; Tanaka, H.; Okuhara, M. J. Antibiot. 1988, 41, 1592.

[4] Sehgal, S. N.; Baker, H.; Vézina, C. J. Antibiot. 1975, 28, 727.

[5] Schwecke, T.; Aparicio, J. F.; Molnar, I.; Konig, A.; Khaw, L. E.; Haydock, S. F.; Oliynyk, M.; Caffrey, P.; Cortes, J.; Lester, J. B.; Bohm, G. A.; Staunton, J.; Leadlay, P. F. Proc. Natl. Acad. Sci. U. S. A. 1995, 92, 7839 .

[6] (a) Motamedi, H.; Shafiee, A. Eur. J. Biochem. 1998, 256, 528. (b) Motamedi, H.; Cai, S. J.; Shafiee, A.; Elliston, K. O. Eur. J.
Biochem. 1997, 244, 74.

[7] Wu, K.; Chung, L.; Revill, W. P.; Katz, L.; Reeves, C. D. Gene 2000, 251,81 .

[8] Mo, S.; Kim, D. H.; Lee, J. H.; Park, J. W.; Basnet, D. B.; Ban, Y. H.; Yoo, Y. J.; Chen, S.-w.; Park, S. R.; Choi, E. A.; Kim, E.; Jin, Y.-Y.; Lee, S.-K.; Park, J. Y.; Liu, Y.; Lee, M. O.; Lee, K. S.; Kim, S. J.; Kim, D.; Park, B. C.; Lee, S.-g.; Kwon, H. J.; Suh, J.-W.; Moore, B. S.; Lim, S.-K.; Yoon, Y. J. J. Am. Chem. Soc. 2011, 133, 976.

[9] Fischbach, M. A.; Walsh, C. T. Chem. Rev. 2006, 106, 3468.

[10] (a) Cortes, J.; Haydock, S. F.; Roberts, G. A.; Bevitt, D. J.; Leadlay, P. F. Nature 1990, 348, 176.

(b) MacNeil, D. J.; Occi, J. L.; Gewain, K. M.; MacNeil, T.; Gibbons, P. H.; Ruby, C. L.; Danis, S. J. Gene 1992, 115, 119.

[11] Hopwood, D. Chem. Rev. 1997, 97, 2465.

[12] (a) Hubbard, B. K.; Walsh, C. T. Angew. Chem., Int. Ed. 2003, 42, 730 .

(b) Lawen, A.; Zocher, R. J. Biol. Chem. 1990, 265, 11355.

[13] Marahiel, M. A.; Stachelhaus, T.; Mootz, H. D. Chem. Rev. 1997, 97, 2651.

[14] Gatto, G. J.; McLoughlin, S. M.; Kelleher, N. L.; Walsh, C. T. Biochemistry 2005, 44, 5993.

[15] (a) Chen, D. D.; Zhang, Q.; Zhang, Q. L.; Cen, P. L.; Xu, Z. N.; Liu, W. Appl. Environ. Microbiol. 2012, 78, 5093.

(b) Mo, S.; Ban, Y. H.; Park, J. W.; Yoo, Y. J.; Yoon, Y. J. J. Ind. Microbiol. Biotechnol. 2009, 36, 1473.

[16] Paiva, N. L.; Roberts, M. F.; Demain, A. L. J. Ind. Microbiol. 1993, $12,423$.

[17] Wallace, K. K.; Reynolds, K. A.; Koch, K.; McArthur, H. A. I.; Brown, M. S.; Wax, R. G.; Moore, B. S. J. Am. Chem. Soc. 1994, 
116, 11600.

[18] Lowden, P. A. S.; Bohm, G. A.; Staunton, J.; Leadlay, P. F. Angew. Chem., Int. Ed. 1996, 35, 2249.

[19] (a) Motamedi, H.; Shafiee, A.; Cai, S. J.; Streicher, S. L.; Arison, B. H.; Miller, R. R. J. Bacteriol. 1996, 178, 5243.

(b) Shafiee, A.; Motamedi, H.; Chen, T. Eur. J. Biochem. 1994, 225,755 .

[20] Lowden, P. A. S.; Wilkinson, B.; Bohm, G. A.; Handa, S.; Floss, H. G.; Leadlay, P. F.; Staunton, J. Angew. Chem., Int. Ed. 2001, 40, 777.

[21] Gregory, M. A.; Gaisser, S.; Lill, R. E.; Hong, H.; Sheridan, R. M.; Wilkinson, B.; Petkovic, H.; Weston, A. J.; Carletti, I.; Lee, H. L.; Staunton, J.; Leadlay, P. F. Angew. Chem., Int. Ed. 2004, 43, 2551.

[22] Molnar, I.; Aparicio, J. F.; Haydock, S. F.; Khaw, L. E.; Schwecke, T.; Konig, A.; Staunton, J.; Leadlay, P. F. Gene 1996, 169, 1.

[23] Andexer, J. N.; Kendrew, S. G.; Nur-e-Alam, M.; Lazos, O.; Foster, T. A.; Zimmermann, A.-S.; Warneck, T. D.; Suthar, D.; Coates, N. J.; Koehn, F. E.; Skotnicki, J. S.; Carter, G. T.; Gregory, M. A.; Martin, C. J.; Moss, S. J.; Leadlay, P. F.; Wilkinson, B. Proc. Natl. Acad. Sci. U. S. A. 2011, 108, 4776.

[24] Knaggs, A. R. Nat. Prod. Rep. 2003, 20, 119.

[25] (a) Bornemann, S.; Theoclitou, M.-E.; Brune, M.; Webb, M. R.; Thorneley, R. N. F.; Abell, C. Bioorg. Chem. 2000, 28, 191.

(b) Du, W.; Wallis, N. G.; Mazzulla, M. J.; Chalker, A. F.; Zhang, L.; Liu, W.-S.; Kallender, H.; Payne, D. J. Eur. J. Biochem. 2000, 267, 222.

(c) Liu, Q.; Li, Y.; Wu, Y.; Yan, H. J. Biomol. NMR 2000, 17, 277.

[26] Gregory, M. A.; Hong, H.; Lill, R. E.; Gaisser, S.; Petkovic, H.; Low, L.; Sheehan, L. S.; Carletti, I.; Ready, S. J.; Ward, M. J.; Kaja, A. L.; Weston, A. J.; Challis, I. R.; Leadlay, P. F.; Martin, C. J.; Wilkinson, B.; Sheridan, R. M. Org. Biomol. Chem. 2006, 4, 3565 .

[27] Byrne, K. M.; Shafiee, A.; Nielsen, J. B.; Arison, B.; Monaghan, R. L.; Kaplan, L. Dev. Ind. Microbiol. 1993, 32, 29.

[28] (a) Kato, Y.; Bai, L. Q.; Xue, Q.; Revill, W. P.; Yu, T. W.; Floss, H. G. J. Am. Chem. Soc. 2002, 124, 5268.

(b) Carroll, B. J.; Moss, S. J.; Bai, L. Q.; Kato, Y.; Toelzer, S.; Yu, T. W.; Floss, H. G. J. Am. Chem. Soc. 2002, 124, 4176.

[29] Yu, T. W.; Bai, L. Q.; Clade, D.; Hoffmann, D.; Toelzer, S.; Trinh, K. Q.; Xu, J.; Moss, S. J.; Leistner, E.; Floss, H. G. Proc. Natl. Acad. Sci. U. S. A. 2002, 99, 7968.

[30] Reeves, C. D.; Chung, L. M.; Liu, Y. Q.; Xue, Q.; Carney, J. R.; Revill, W. P.; Katz, L. J. Biol. Chem. 2002, 277, 9155.

[31] Dorrestein, P. C.; Van Lanen, S. G.; Li, W.; Zhao, C.; Deng, Z.; Shen, B.; Kelleher, N. L. J. Am. Chem. Soc. 2006, 128, 10386.

[32] Watanabe, K.; Khosla, C.; Stroud, R. M.; Tsai, S. C. J. Mol. Biol. 2003, 334, 435 .

[33] (a) Chen, H. W.; Thomas, M. G.; O'Connor, S. E.; Hubbard, B. K.;
Burkart, M. D.; Walsh, C. T. Biochemistry 2001, 40, 11651.

(b) Chen, H. W.; Walsh, C. T. Chem. Biol. 2001, 8, 301

[34] Chan, Y. A.; Boyne, M. T., II; Podevels, A. M.; Klimowicz, A. K.; Handelsman, J.; Kelleher, N. L.; Thomas, M. G. Proc. Natl. Acad. Sci. U. S. A. 2006, 103, 14349.

[35] Paiva, N. L.; Demain, A. L.; Roberts, M. F. J. Nat. Prod. 1991, 54, 167.

[36] Goranovic, D.; Kosec, G.; Mrak, P.; Fujs, S.; Horvat, J.; Kuscer, E.; Kopitar, G.; Petkovic, H. J. Biol. Chem. 2010, 285, 14292.

[37] Paiva, N. L.; Demain, A. L.; Roberts, M. F. Enzyme Microb. Technol. 1993, 15, 581.

[38] Khaw, L. E.; Bohm, G. A.; Metcalfe, S.; Staunton, J.; Leadlay, P. F. J. Bacteriol. 1998, 180, 809.

[39] Gatto, G. J.; Boyne, M. T.; Kelleher, N. L.; Walsh, C. T. J. Am. Chem. Soc. 2006, 128, 3838.

[40] Shafiee, A.; Motamedi, H.; Dumont, F. J.; Arison, B. H.; Miller, R. R. J. Antibiot. 1997, 50, 418.

[41] Maddess, M. L.; Tackett, M. N.; Ley, S. V. In Natural Compounds as Drugs, Vol. 66, Eds.: Petersen, F.; Amstutz, R., Birkhäuser Basel, Berlin, 2008, p. 13.

[42] (a) Jones, T. K.; Mills, S. G.; Reamer, R. A.; Askin, D.; Desmond, R.; Volante, R. P.; Shinkai, I. J. Am. Chem. Soc. 1989, 111, 1157; (b)Jones, T. K.; Reamer, R. A.; Desmond, R.; Mills, S. G. J. Am. Chem. Soc. 1990, 112, 2998.

[43] Nakatsuka, M.; Ragan, J. A.; Sammakia, T.; Smith, D. B.; Uehling, D. E.; Schreiber, S. L. J. Am. Chem. Soc. 1990, 112, 5583.

[44] (a) Ireland, R. E.; Gleason, J. L.; Gegnas, L. D.; Highsmith, T. K. J. Org. Chem. 1996, 61, 6856.

(b) Ireland, R. E.; Liu, L.; Roper, T. D. Tetrahedron 1997, 53, 13221 .

(c) Ireland, R. E.; Liu, L.; Roper, T. D.; Gleason, J. L. Tetrahedron 1997, 53, 13257.

[45] Gu, R.-L.; Sih, C. J. Tetrahedron Lett. 1990, 31, 3287.

[46] Jones, A. B.; Villalobos, A.; Linde, R. G.; Danishefsky, S. J. J. Org. Chem. 1990, 55, 2786.

[47] Smith, A. B.; Chen, K.; Robinson, D. J.; Laakso, L. M.; Hale, K. J. Tetrahedron Lett. 1994, 35, 4271.

[48] Kosec, G.; Goranovic, D.; Mrak, P.; Fujs, S.; Kuscer, E.; Horvat, J.; Kopitar, G.; Petkovic, H. Metab. Eng. 2012, 14, 39.

[49] Lechner, A.; Wilson, M. C.; Ban, Y. H.; Hwang, J.-y.; Yoon, Y. J.; Moore, B. S. ACS Synth. Biol. DOI: 10.1021/sb3001062.

[50] (a) Gregory, M. A.; Petkovic, H.; Lill, R. E.; Moss, S. J.; Wilkinson, B.; Gaisser, S.; Leadlay, P. F.; Sheridan, R. M. Angew. Chem., Int. Ed. 2005, 44, 4757.

(b) Goss, R. J. M.; Lanceron, S. E.; Wise, N. J.; Moss, S. J. Org. Biomol. Chem. 2006, 4, 4071.

(c) Lowden, P. A. S.; Böhm, G. A.; Metcalfe, S.; Staunton, J.; Leadlay, P. F. ChemBioChem 2004, 5, 535. 